Proyecciones Journal of Mathematics Vol. 33, N ${ }^{o}$ 1, pp. 13-23, March 2014.

Universidad Católica del Norte

Antofagasta - Chile

\title{
Some characterization theorems on dominating chromatic partition-covering number of graphs
}

\author{
L. Benedict Michael Raj \\ St. Joseph's College, India \\ and \\ S. K. Ayyaswamy \\ Sastra University, India \\ Received : July 2011. Accepted : January 2013
}

\begin{abstract}
Let $G=(V, E)$ be a graph of order $n=|V|$ and chromatic number $(\mathcal{G})$. A dominating set $D$ of $G$ is called a dominating chromatic partition-cover or dcc-set, if it intersects every color class of every $\mathcal{X}$-coloring of $G$. The minimum cardinality of a dcc-set is called the dominating chromatic partition-covering number, denoted $d c c(G)$. The dcc-saturation number equals the minimum integer $k$ such that every vertex $v \in V$ is contained in a dcc-set of cardinality $k$. This number is denoted by $\operatorname{dccs}(G)$. In this paper we study a few properties of these two invariants $\operatorname{dcc}(G)$ and $\operatorname{dccs}(G)$.
\end{abstract}

Keywords : Dominating set, chromatic partition, dominating chromatic partition-covering number.

Sbject classification : $05 C 69$. 


\section{Introduction, terminology and definitions}

By a graph $G=(V, E)$ we mean a finite, undirected graph without loops or multiple edges, of order $n=|V|$ and size $m=|E|$. For graph theoretic terminology not given here, the reader is referred to Harary [5].

One of the fastest growing areas within graph theory is the study of domination and related subset problems, such as independence, covering and matching. A comprehensive treatment of the fundamentals of domination is given in the book by Haynes et al. [8]. Surveys of several advanced topics in domination can be seen in the book edited by the same authors [7]. Perhaps the most studied area of graph theory is the study of graph colorings, or partitions of either $V$ or $E$ according to certain rules. Topics lying in the intersection of these two areas are starting to appear in the literature, such as partitions of $V$ into dominating sets and the corresponding invariant called the domatic number (cf. Chapter 13 by Zelinka in [7]. This paper is a contribution to this intersection.

We will need several definitions. A set $S \subseteq V$ is called a dominating set if every vertex in $V-S$ is adjacent to a vertex in $S$. The minimum cardinality of a dominating set in $G$ is called the domination number and is denoted by $\gamma(G)$; also a dominating set of minimum cardinality is called a $\gamma$-set. A set $S \subset V$ is called independent if no two vertices in $S$ are adjacent. A $k$-coloring of $G$ is a partition $\pi=\left\{V_{1}, V_{2}, \ldots, V_{k}\right\}$ of $V$ into $k$ independent sets, called color classes. The chromatic number of $G$, denoted $(\mathcal{G})$, equals the minimum integer $k$ such that $G$ has a $k$-coloring. A chromatic partition is a partition $\pi=\left\{V_{1}, V_{2}, \ldots, V_{k}\right\}$ of $V$ into $(\mathcal{G})$ independent sets, or equivalently, a chromatic partition is a $\mathcal{X}$-coloring.

We say that a set $S \subset V$ covers a partition $\pi$ if $S \cap V_{i} \neq \emptyset$ for every $i$, $1 \leq i \leq k$. A chromatic partition cover is a set $S$ that covers every chromatic partition of $G$. If $S$ is also a dominating set, then $S$ is called a dominating chromatic partition-cover of $G$, or a $d c c$-set. The minimum cardinality of a $d c c$-set is called the dominating chromatic partition-covering number, denoted $\operatorname{dcc}(G)$. The concept of a dominating chromatic-partition cover was first introduced and studied by the present authors in [3], who called $d c c(G)$ the chromatic transversal domination number. But since this is not truly a domination number, we have changed the terminology and notation to better reflect the fact these sets are covers (or transversals), but restricted to dominating sets, of the chromatic partitions of a graph. This paper is a continuation of that study.

A vertex $v \in V$ is called $\mathcal{X}$-critical if $(\mathcal{G}-\sqsubseteq)<(\mathcal{G})$. We call this vertex 
a $\mathcal{X}$-critical vertex. If every vertex $v \in V$ is $\mathcal{X}$-critical, then $G$ is called a $\mathcal{X}$-critical graph. It is easy to see, for example, that every complete graph $K_{n}$ and every cycle $C_{2 n+1}$ of odd length is a $\mathcal{X}$-critical graph.

\section{Example 1:}

For the above graph $G, S=\{c, d, e\}$ is a $d c c$-set, since $S$ covers every $\mathcal{X}$-partition of $G$ and so $\operatorname{dcc}(G)=3$.

Example 2: Consider any cycle $C_{n}$ of odd length $n$. As every vertex $v$ of $C_{n}$ is a $\mathcal{X}$-critical vertex, it follows that $\{v\}$ is a colour class of some $\mathcal{X}$-partition of $C_{n}$. Therefore $d c c\left(C_{n}\right)=n$.

A $d c c$-set $S$ is called minimal if no proper subset of $S$ is also a $d c c$-set. The property of being a $d c c$-set is super-hereditary since any superset of a $d c c$-set is also a $d c c$-set. Thus, a set $S$ is a minimal $d c c$-set if and only if for every $v \in S, S-\{v\}$ is not a $d c c$-set.

In [1], Acharya introduced the concept of the domsaturation number $d s(G)$, that equals the minimum integer $k$ such that every vertex $v \in V$ is contained in a dominating set of cardinality $k$. Notice that for any 
graph $G$, either every vertex $v \in V$ is contained in a $\gamma$-set, in which case $d s(G)=\gamma(G)$, or can be added to a $\gamma$-set, in which case $d s(G)=\gamma(G)+1$. Thus, $\gamma(G) \leq d s(G) \leq \gamma(G)+1$.

A dominating set $S$ of a graph $G$ is called a global dominating set if $S$ is also a dominating set of the complement $\bar{G}$ of $G$. The minimum cardinality of a global dominating set, $\gamma_{g}(G)$, is called the global domination number of $G$. The global domsaturation number $\operatorname{dsg}(G)$ equals the minimum integer $k$ such that every vertex $v$ is contained in a global dominating set of cardinalilty $k$ [7].

Finally, The open neighborhood $N(v)$ of a vertex $v \in V$ equals the set of vertices adjacent to $v$ in $G$, that is, $N(v)=\{u \mid u v \in E\}$. The closed neighborhood $N[v]$ of a vertex $v \in V$ is the set $N(v) \cup\{v\}$. Let $S \subseteq V$ be a set of vertices and let $u \in S$. We define $p n[u, S]=N[u]-N[S-u]$ and $p n(u, S)=N(u)-N(S-u)$.

The set of private neighbors of a vertex in $u \in S$ is denoted by $p n[u, S]$. Notice that if a vertex $u \in S$ is not adjacent to any vertex in $S$, then $u \in p n[u, S]$, in which case we say that $u$ is its own private neighbor, while every other private neighbor of $u$ is a vertex in $V-S$.

The following are the important results proved in [3] by the authors.

Theorem 1.1. ([3,9]) A dcc-set $S$ is minimal if and only if for every vertex $u \in S$, at least one of the following holds. (i) $p n[u, S] \neq \emptyset$. (ii) There exists a chromatic partition $\pi=\left\{V_{1}, V_{2}, \ldots, V_{k}\right\}$ such that $S \cap V_{i}=\{u\}$ for some $i$.

Theorem 1.2. ([3]) For a connected graph $G, d c c(G)=n$ if and only if $G$ is $\mathcal{X}$-critical.

Theorem 1.3. ([3]) Let $G$ be a connected bipartite graph of order $n \geq 3$ and vertex bipartition $(X, Y)$ with $|X| \leq|Y|$. Then $d c c(G)=\gamma(G)+1$ if and only if every vertex in $X$ has at least two leaves as its neighbors in $Y$. 
Theorem 1.4. ([3]) For any graph $G$, (i) $\gamma(G) \leq \gamma_{g}(G) \leq d c c(G)$. (ii) $(\mathcal{G}) \leq$ $\operatorname{dcc}(\mathcal{G})$.

Result $1.5([3])$.

(i) $\operatorname{dcc}\left(P_{n}\right)=\gamma\left(P_{n}\right)=\left\lceil\frac{n}{3}\right\rceil, n \geq 4$.

(ii) Every $\mathcal{X}$ - critical vertex is in every $d c c$-set.

Theorem 1.6 ([8]). If $G$ is a graph without isolated vertices, then $\gamma(G) \leq$ 2 .

Theorem 1.7([2]) For a connected bipartite graph $G$ of order $n \geq 3$, $d s g(G)=d s(G)$.

Result 1.8 ([3]).

(i) $d c c\left(K_{n}\right)=d c c\left(\overline{K_{n}}\right)=n$ and $d c c\left(K_{m, n}\right)=2$.

(ii) Let $G$ be a connected bipartite graph with vertex bipartition $(X, Y)$. If there exists a $\gamma$-set $S$ of $G$ such that $S \cap X \neq \emptyset$, then $d c c(G)=\gamma(G)$, otherwise $d c c(G)=\gamma(G)+1$. In particular $d c c\left(C_{n}\right)=\left\lceil\frac{n}{3}\right\rceil$, if $n$ is even.

(iii) If every vertex $v$ of a graph $G$ forms a color class of some chromatic partition of $G$, then $d c c(G)=n$. In particular $d c c\left(C_{n}\right)=n$, if $n$ is odd and

$$
\operatorname{dcc}\left(W_{n}\right)=\left\{\begin{array}{c}
n, \text { if } n \text { is even; } \\
3, \text { if } n \text { is odd }
\end{array}, \text { where } W_{n}\right. \text { is a whell with }
$$

(iv) $d c c(P)=5$, where $P$ is the Petersen graph.

Theorem 1.9 ([3]). Let $G$ be a connected graph with bipartition $(X, Y)$ where $|X| \leq|Y|$ and $n \geq 3$. Then $\operatorname{dcc}(G)=\gamma(G)+1$ if and only if every vertex in $X$ has at least two neighbours which are leaves. 


\section{Main Results}

Analogous to $d s(G)$ and $d s g(G)$ in [2] we define a number $d c c s(G)$ using which we can characterize graphs for which $\operatorname{dcc}(G)=n-1$.

Definition 2.1. Let $G$ be a graph. The $d c c$-saturation number of a vertex $v$, denoted by $\operatorname{dccs}(v)$, equals the minimum integer $k$ such that the vertex $v$ is contained in a $d c c$-set of cardinality $k$ and $d \operatorname{ccs}(G)=\max \{d \operatorname{ccs}(v): v \in V\}$.

Remark 2.2. (i) If $S$ is a dcc-set, then for any $u \in V-S, S \cup\{u\}$ is a $d c c$-set and hence $d c c(G) \leq d c c s(G) \leq d c c(G)+1$.

(ii) For every $v \in V, \operatorname{dcc}(G) \leq \operatorname{dccs}(v) \leq \operatorname{dccs}(G)$.

(iii) $d s(G) \leq d s g(G) \leq d c c s(G)$.

Definition 2.3. A graph $G$ is said to be in class I or class II according as $d c c s(G)=d c c(G)$ or $d c c s(G)=d c c(G)+1$.

It can be verified that the following graphs belong to class I:-

(i) All $\mathcal{X}$-critical graphs, in particular $K_{n}$, for every $n$ and the wheel $W_{n}$, $n$ even.

(ii) The cycle $C_{n}$, for every $n$.

(iii) The Petersen graph in view of a theorem given in [3].

Proposition 2.4. For $n \geq 4, P_{n}$ is a class $I$ graph if and only if $n \equiv 1$ $(\bmod 3)$.

Proof. Clearly $P_{4}$ is a class I graph. Let $n \geq 5$. If $n \not \equiv 1(\bmod 3)$, then by a Theorem in [2], the domsaturation number $d_{s}\left(P_{n}\right)=\gamma\left(P_{n}\right)+1$ and consequently $\operatorname{dccs}\left(P_{n}\right) \geq d_{s}\left(P_{n}\right)=\operatorname{dcc}\left(P_{n}\right)+1$. Hence $P_{n}$ is a class II graph.

Conversely, suppose $n \equiv 1 \quad(\bmod 3)$. Let $n=3 k+1 ; k \geq 2$. Let the vertices of $P_{n}$ be $\{1,2, \ldots, 3 k+1\}$. Then $D_{1}=\{1,3,6, . ., 3(k-1), 3 k\}$, $D_{2}=\{2,5, \ldots, 3 k-1,3 k\}$ and $D_{3}=\{1,4,7, \ldots, 3 k-2,3 k+2\}$ are dcc-sets and so $\operatorname{dccs}\left(P_{n}\right)=\operatorname{dcc}(G)$.

Proposition 2.5. Let $H$ be a connected graph with $n \geq 3$. Then the corona $H \circ K_{1}$, is a class $I$ graph if and only if $H$ has no critical vertex. 
Proof. To prove this result, we prove that $G$ is a Class II graph if and only if $H$ has a critical vertex.

Let $\operatorname{dccs}(G)=d c c(G)+1$. Then $d c c s(v)=d c c(G)=|V(H)|$ for every $v \in V(H)$. As $d c c s\left(H \circ K_{1}\right)=d c c\left(H \circ K_{1}\right)+1$, there exists a leaf $v_{0}$ for which $d c c s\left(v_{0}\right)=d c c\left(H \circ K_{1}\right)+1$. Let $u_{0}$ be the support of $v_{0}$. Then $u_{0}$ is a critical vertex; otherwise $\left(V(H)-\left\{u_{0}\right\}\right) \cup\left\{v_{0}\right\}$ becomes a dcc-set containing $v_{0}$ of cardinality $\operatorname{dcc}(G)$.

Conversely, if $u_{0}$ is a critical vertex of $H$, then $u_{0}$ is also a critical vertex of $G$ and so $d c c s\left(v_{0}\right)=d c c(G)+1$ where $v_{0}$ is a leaf having $u_{0}$ as its support. So $G$ is a class II graph.

Lemma 2.6. For a connected graph $G, \operatorname{dccs}(G)=n$ if and only if $G$ has at most one vertex that is not $\mathcal{X}$-critical.

Proof. If $\operatorname{dccs}(G)=n$, then $d c c(G)=n$ or $n-1$. If $d c c(G)=n$, then by Theorem 1.2, $G$ becomes a $\mathcal{X}$-critical graph with $n$ vertices.

Let $d c c(G)=n-1$. Let $S$ be a $d c c$-set of $G$. Then, $S=V-\{u\}$ for some $u \in V$. Clearly $u$ is not a critical vertex. As $d \operatorname{ccs}(G)=n$, we have $d \operatorname{ccs}(u)=n$. Suppose $w \neq u$ is not critical, then $V-\{w\}$ is a dcc-set containing $u$, contradicting the fact $d \operatorname{ccs}(u)=n$.

Conversely, if all the vertices of $G$ are critical vertices, then by Theorem 1.3, $d c c(G)=n=d c c s(G)$. If there exists a unique vertex $u$ that is not critical, then $V-\{u\}$ is the only $d c c$-set in $G$ and so $d c c s(G)=n$.

Lemma 2.7. For a connected graph $G, d c c(G)=d c c s(G)=n-1$ if and only if $G$ has exactly two vertices $u$ and $v$ that are not critical and they satisfy at least one of the following conditions:

(a) one is a support and the other is its only adjacent leaf.

(b) $\chi(G-\{u, v\})<(\mathcal{G})$. 
Proof. Let $G$ be a connected graph of order $n \geq 2$ with $\operatorname{dcc}(G)=$ $\operatorname{dccs}(G)=n-1$. Obviously $(\mathcal{G}) \geq \in$. As $d c c(G)=n-1$, by Theorem 1.2, $G$ is not a $\mathcal{X}$-critical graph. Let $H$ be a $\mathcal{X}$-critical subgraph of $G$ of maximum size. Let $|V(H)|=k$. As $(\mathcal{H})=(\mathcal{G}), V(H)$ is a chromatic partition cover of $G$. So $n-1=d c c(G) \leq k+n-k 2 \leq \frac{n+k}{2}$. This implies that $n \leq k+2$. Now $n \neq k$, for otherwise $G$ becomes a $\mathcal{X}$-critical graph. So $n=k+2$ or $k+1$.

Case 1: Let $n=k+2$. Let $V(G)-V(H)=\{u, v\}$. Suppose $u$ and $v$ are non-adjacent or both are not leaves, then $V(H)$ becomes a $d c c$-set of $G$, a contradiction to $\operatorname{dcc}(G)=n-1$. Therefore $u$ and $v$ are adjacent and one of them, say $v$, is a leaf. Every vertex $w$ distinct from $u$ and $v$ are critical vertex of $G$, for otherwise $d c c(G)=p-2$. So (a) is true in this case.

Case 2: Let $n=k+1$. When $(\mathcal{G})=\in$, we have $G \simeq P_{3}$ satisfying (b). Assume that $(\mathcal{G}) \geq \ni$.

Then $k \geq 3$. Let $V(G)-V(H)=\{v\}$. By definition of $H, H=G-\{v\}$. As $\operatorname{dccs}(G)=n-1$, there exists a vertex $u$ in $H$ such that $V(G)-\{u\}$ is a $d c c$-set of $G$ containing $v$. Obviously $u$ and $v$ are not critical vertices of $G$.

\section{Claim:}

item[(i)] No vertex in $G$ is a leaf. item[(ii)] $G-u$ is a $\mathcal{X}$-critical subgraph of $G$. item[(iii)] $u$ and $v$ are not adjacent in $G$. item[(iv)] $N(u) \cup N(v)=V(G)$. item[(v)] $u$ and $v$ are the only vertices in $G$ that are not critical.

$v$ cannot be a leaf, for otherwise $\operatorname{dccs}(v)=n$. Since $H$ is a $\mathcal{X}$-critical subgraph, no vertex of $H$ is a leaf in $G$. Hence (i) is true. Suppose (ii) is not true. Then there exists a vertex $w$ in $G-\{u\}$ such that $(\mathcal{G}-\{\sqcap, \sqsupseteq\})=$ $(\mathcal{G}-\sqcap)=(\mathcal{G})$. Hence $V-\{u, w\}$ becomes a $d c c$-set in view of (i), a contradiction.

Suppose $u$ and $v$ are adjacent. Then as $u$ and $v$ are not critical vertices, the set $V-\{u, v\}$ is a $d c c$-set by (i), a contradiction and so (iii) is true. Next we prove that $N(u) \cup N(v)=V(G)$. Suppose $w \notin N(u) \cup N(v)$, then $(\mathcal{G}-\{\sqsubseteq, \sqsupseteq\})=(\mathcal{G})-\infty$ as $G-v$ is a $\mathcal{X}$-critical subgraph of $G$. Similarly $\chi(G-\{u, w\})=(\mathcal{G})-\infty$. This implies that $u, v, w$ belong to every $d c c$-set of $G$, a contradiction to the fact that $V-\{u\}$ is a $d c c$-set containing $v$. This proves (iv). 
Suppose $w \neq u, v$, such that $w$ is not a critical vertex of $G$. By (iv) $w$ is adjacent to either $u$ or $v$, say $u$. As $u, v, w$ are not critical vertices, none of the sets $\{u\},\{v\},\{w\}$ will form a color class for any chromatic partition of $G$. Since $u$ and $w$ are adjacent, they belong to different color classes of every chromatic partition of $G$. This implies that $V-\{u, v\}$ is a $d c c$ set of $G$ by (i), a contradiction. If $\{u, v\}$ is not a color class of any chromatic partition of $G$, then $V(G)-\{u, v\}$ is a $d c c$-set, a contradiction. So $\chi(G-\{u, v\})<(\mathcal{G})$ satisfying $(\mathrm{b})$.

The converse is easily proved.

Theorem 2.8. For a connected graph $G, d c c(G)=n-1$ if and only if $G$ has exactly one vertex that is not critical or two vertices $u$ and $v$, both are not critical vertices satisfying any one of the following properties:

(i) One is a support and the other is its adjacent leaf.

(ii) $\chi(G-\{u, v\})<(\mathcal{G})$.

Proof. If $d c c(G)=n-1$, then $\operatorname{dccs}(G)=n-1$ or $n$. So by Lemmas 2.6 and 2.7 , we get the required result.

Corollary 2.9. If $G$ is a connected bipartite graph, then $\operatorname{dcc}(G)=n-1$ if and only if $G \simeq P_{3}$.

Proof. Let $d c c(G)=n-1$. Obviously $n \geq 3$. When $n>4$ by Theorem 2.8, $G$ will have at least two critical vertices which is impossible. So $G \simeq P_{3}$. The converse in obvious.

Theorem 2.10. For a connected bipartite graph $G\left(\neq K_{2}\right), d s(G)=d c c s(G)$. 
Proof. If $G=K_{1, n-1}$, then $d s(G)=2=d c c(G)$. Therefore assume that $G \neq K_{1, n-1}$. Then every dominating set has vertices from each partition set. That means, every dominating set is also a chromatic partition cover and hence $\operatorname{dcc}(G) \leq \gamma(G)$. Since $\gamma(G) \leq d c c(G)$ is evident, we have that $d c c(G)=\gamma(G)$. Hence $\gamma(G) \leq d s(G) \leq d c c(G)+1=\gamma(G)+1$. Therefore, if $d s(G) \neq d \operatorname{dccs}(G)$, then $d s(G)=\gamma(G)$ and $d c c s(G)=\gamma(G)+1$. From the first equality follows that every vertex lies on a minimum dominating set. From the second that there is a vertex that does not lie on a minimum $d c c$-set or rather (since $d c c(G)=\gamma(G)$ ) on a minimum dominating set. Hence, a contradiction and $d c(G)=\operatorname{dccs}(G)$ follows.

The classification theorem for $d s g(G)=d s(G)$ given in [2] follows as a corollary of Theorem 2.10 .

Corollary 2.11. For a connected bipartite graph $G\left(\neq K_{2}\right), d s g(G)=d s(G)=$ $\operatorname{dccs}(G)$.

Proof. Since $d s(G) \leq d s g(G) \leq d c c s(G)$, the result follows from Theorem 2.10.

\section{References}

[1] B. D. Acharya, The Strong Domination Number of a Graph and Related Concepts, Jour. Math. Phy. Sci. 14, no. 5, pp. 471-475, (1980).

[2] S. Arumugam and R. Kala, Domsaturation Number of a Graph, Indian J. Pure. Appl. Math. 33, no. 11, pp. 1671-1676, (2002).

[3] L. Benedict Michael Raj, S. K. Ayyaswamy, and S. Arumugam, Chromatic Transversal Domination in Graphs, J. Comb. Math. Comb. Computing, Vol. 75, pp. 33-40, (2010).

[4] G. Chartrand and P. Zang, Chromatic Graph Theory, CRC Press, Boca Raton, FL, (2009).

[5] F. Harary, Graph Theory, Addison-Wesley, Reading, Mass, (1969). 
[6] F. Harary, S. Hedetneimi and R. Robinson, Uniquely Colorable Graphs, J. Combin. Theory 6, pp. 264-270, (1969).

[7] T. W. Haynes, S. T. Hedetniemi, and P. J. Slater, Domination in Graphs: Advanced Topics, Marcel Dekker Inc., (1998).

[8] , Fundamentals of Domination in Graphs, Marcel Dekker Inc., (1998).

[9] O. Ore, Theory of Graphs, Amer. Math. Soc. Colloq. Publ. 38, (Amer. Math Soc., Providence, RI), (1962).

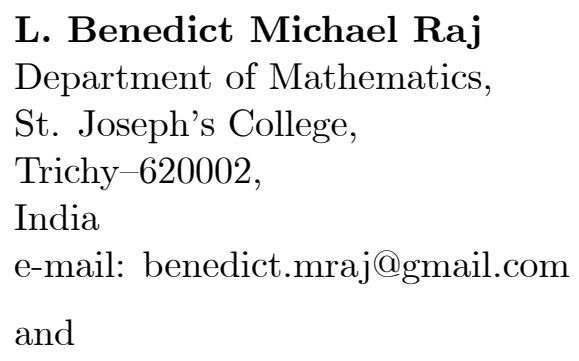

